\title{
Non-Invasive Multiparametric Monitoring through Measurement of the Optic Nerve Sheath Diameter and Transcranial Doppler Ultrasound as an Approach to Cerebral Hemodynamics
}

Anselmo A. Abdo-Cuza ${ }^{1}$, Roberto Castellanos-Gutierrez ${ }^{1}$, Juan A. Gutiérrez-Martínez ${ }^{1}$, Juliette Suárez-López ${ }^{2}$, Geydy Leal-Alpizar ${ }^{1}$, Yalina Benitez-Quevedo ${ }^{1}$, Francisco Gomez-Peire ${ }^{1}$, Juan C Lopez-Gonzalez ${ }^{1}$, Rafael Machado-Martinez ${ }^{1}$, Jonathan Pi-Ávila1 ${ }^{1}$, Daniel González-González ${ }^{1}$.

${ }^{1}$ Centro de Investigaciones Médico Quirúrgicas, La Habana, Cuba.

${ }^{2}$ Hospital Hermanos Ameijeiras, La Habana, Cuba.

aaabdo@infomed.sld.cu

*Corresponding Author: Anselmo A. Abdo Cuza.Unidad de Cuidados Intensivos. Centro de Investigaciones Médico Quirúrgicas, La Habana, Cuba.

\section{Abstract}

Among a group of monitoring techniques that estimate ICP non-invasively, the measurement of the optic nerve sheath diameter (ONSD) seems to be the most promising. The prediction threshold to correlate with a high ICP ( $>20 \mathrm{mmHg}$ ) has been proposed by some authors between 5.7 and $5.9 \mathrm{~mm}$ but there is no uniform cut-off point in the literature.

Objective: To determine the utility of the ONSD measurement as an estimate of non-invasive ICP; correlating it with invasive measurements and cerebral hemodynamic patterns by transcranial Doppler.

Method: The sample consisted of a series of five neurocritical cases admitted to the intensive care unit of the Centro de Investigaciones Médico Quirúrgicas, to which an intraventricular catheter was placed to measure ICP by air bag using the Spiegelberg monitor. Each patient underwent simultaneous measurements of ICP, transcranial Doppler ultrasound and retrobulbar ultrasound to measure ONSD. In total, 16 measurements were made that integrated the data for statistical analysis.

Results: The value of ONSD over which there was ICP $\geq 20 \mathrm{mmHg}$ was $6.50 \mathrm{~mm}$, constituting the cut-off point. The correlation between mVMCA and ONSD was moderate and inverse, with statistical significance $(r=-0.532$, $p=0.034$ ).

Conclusions: Non-invasive multiparametric monitoring as part of critical ultrasound shows promise in neurocritical patients.

\section{INTRODUCTION}

Intracranial pressure (ICP) integrates the group of parameters that constitute multiparametric neuro monitoring, a fundamental pillar in the care of neurocritical patients [1]. The invasive method through catheters in intraventricular or intraparenchymal locations is the reference technique or gold standard [2]. The procedure has a group of limitations such as the possibility of infectious complications (1 - 10\%), hemorrhagic complications (0.8 - $3 \%)$, in addition to requiring the material and professional resources, plus the time necessary for the realization, that in emergency situations it is vital [3].

Among a group of monitoring techniques that estimate ICP non-invasively, the measurement of the optic nerve sheath diameter (ONSD) seems to be the most promising [4]. 
The optic nerve as a structure of the central nervous system is surrounded by cerebrospinal fluid (CSF) and by meningeal covers that form the sheath of the optic nerve. Hansen and Helmke [5] in 1997 described variations in the ONSD in accordance with changes in the ICP; because the pressure increase is transmitted to the CSF of the subarachnoid space limited by the optic nerve sheath, widening it.

The ONSD prediction threshold to correlate with a high ICP (> $20 \mathrm{mmHg}$ ) has been proposed by some authors between 5.7 and $5.9 \mathrm{~mm}$ but there is no uniform cutoff point in the literature [6].

Preliminary experiences of the authors, related to findings of ONSD measurements above the reference values shown in the literature, in patients with no clinical evidence of increased ICP, motivated the design of the present investigation with the objective of determining the utility of the technique as an estimate of ICP in a non-invasive way; correlating it with invasive measurements and cerebral hemodynamic patterns by transcranial Doppler (TCD).

\section{METHOD}

The sample consisted of a series of five neurocritical cases admitted to the intensive care unit of the Centro de InvestigacionesMédicoQuirúrgicas during 2017 and to which an intraventricular catheter (3TT catheter) was placed for measurement of ICP by air bag in Spiegelberg monitor, Figure 1.

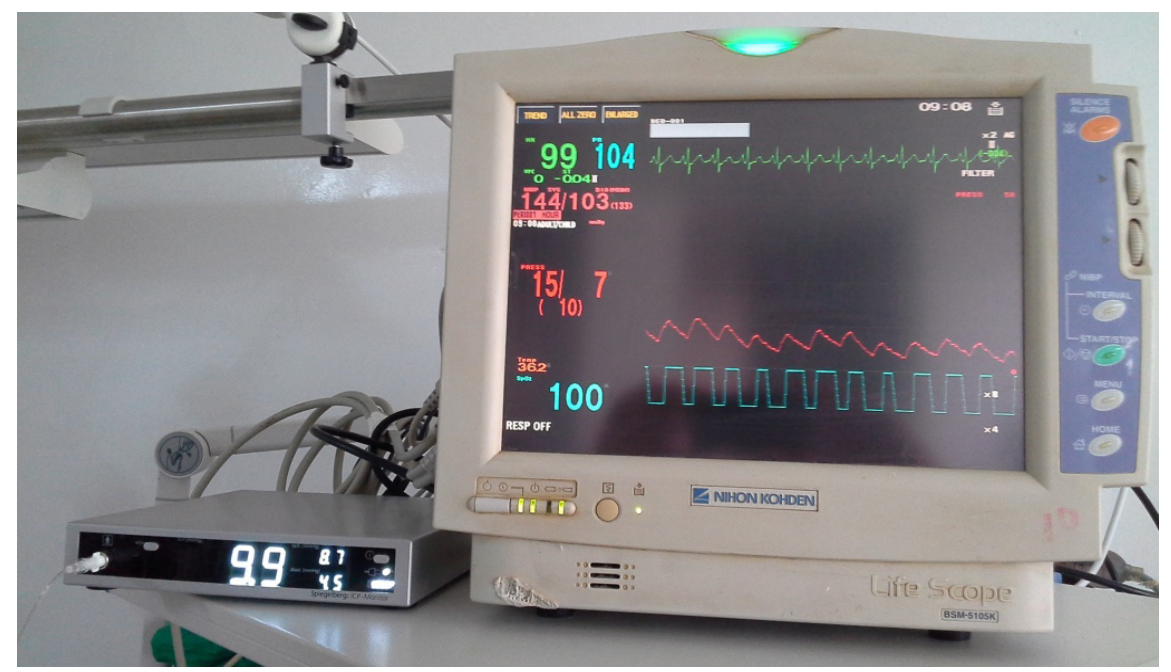

Fig1. Spiegelberg monitor (on the left) showing the measured intracranial pressure (ICP) with intraventricular Spiegelberg 3TT catheter by air bag. On the right, a multiparameter monitor is shown where the ICP curve is displayed along with other systemic monitoring parameters.

The general data of the cases of the series are presented in Table 1.

Table 1. General data of the case series

\begin{tabular}{|c|c|c|c|}
\hline Patient & Gender & Age & Main diagnosis \\
\hline 1 & Male & 58 & Postoperative brain tumor \\
\hline 2 & Female & 64 & Subarachnoid hemorrhage \\
\hline 3 & Male & 70 & Postoperative brain tumor \\
\hline 4 & Male & 76 & Subarachnoid hemorrhage \\
\hline 5 & Female & 50 & Subarachnoid hemorrhage \\
\hline
\end{tabular}

Each patient underwent simultaneous measurements of ICP, TCD ultrasound and retrobulbar ultrasound to measure ONSD. In total, 16 measurements were made that integrated the data for statistical analysis.

\section{Transcranial Doppler Ultrasound}

The transcranial Doppler sonographic study was performed with the patient in the supine position using a device DWL (ElektronischeSysteme GmbH, Germany) Multidop T. Insonation of the vessels to be examined was performed with a $2 \mathrm{MHz}$ pulsed Doppler transducer. The sample volume, gain and power remained constant during the investigation. Through the temporal window bilaterally sonograms of the right and left middle cerebral artery (MCA) were obtained in depths between 
Measurement of the Optic Nerve Sheath Diameter and Transcranial Doppler Ultrasound as an Approach to Cerebral Hemodynamics

45-55 mm. Flow velocity (fv) and pulsatibility index (PI) were recorded. It was considered that interhemispheric asymmetries existed between the fv of MCA if the difference was greater than $20 \%$. The distal extracranial portion of the internal carotid artery (eICA) was examined through the submandibular windows at a depth between 30-55 $\mathrm{mm}$, with the $2 \mathrm{MHz}$ transducer in the pulsed Doppler mode.

None of the patients presented decreases in their hematocrit, no significant blood gas alterations, limits of arterial oxygen pressure (PO2) accepted, between 60 and $110 \mathrm{mmHg}$, limits of pressure of carbon dioxide (PCO2) accepted, between 35 and $45 \mathrm{mmHg}$, nor elevations of its body temperature above $38^{\circ} \mathrm{C}$ at the time of performing the TCD, requiring an mean arterial pressure (MAP) greater than $65 \mathrm{mmHg}$.

\section{The Cerebral Hemodynamic Patterns were} Defined as Follows [7]

Normal: one that presented a cerebral blood flow
(CBF) with mean velocity (mv) and PI within the normality range defined in the reference values.

Low Flow: the one that presented an CBF with mv lower than the reference values, independently of the PI, Figure 2.

High Resistance: the one that presented an CBF with PI greater than the reference values, with mv within normality.

Vasospasm: the one that presented an CBF with mv greater than the reference values, with a Lindegaard index $>3$, regardless of the PI.

Hyperemia: the one that presented an CBF with mv greater than the reference values, with a Lindegaard index $<3$, regardless of the PI. The Lindegaard index is the quotient between the mvMCA and the mv eICA.

Cerebral Circulatory Arrest: one that presented findings of isolated systolic spikes or reverberant flow.

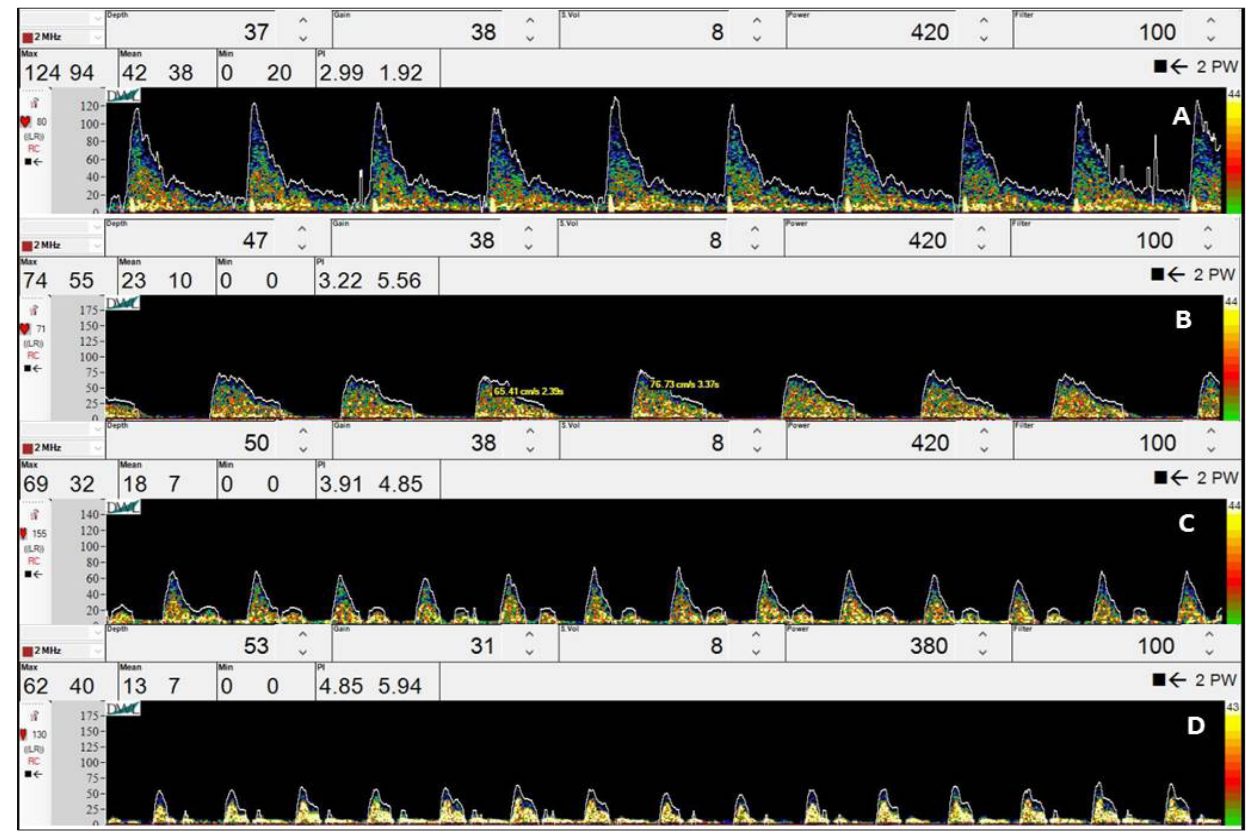

Fig 2. Doppler spectra of the middle cerebral artery (MCA), obtained in a transtemporal window at depths between 37 - $53 \mathrm{~mm}$. From A to D, there is a decrease in mean cerebral blood flow velocities (Mean) and an increase in the pulsatility index (PI), patterns compatible with hypoperfusion or low cerebral flow.

Retrobulbar ultrasound for measurement of optic nerve sheath diameter. For the ONSD measurement, a 3-8 MHz linear transducer was used. The patients were examined in supine. The transducer was placed in axial plane on the temporal portion of the closed upper eyelid using a thick layer of ultrasound gel. In this way, the retrobulbar part of the optic nerve could be visualized in an axial plane that showed the papilla and the optic nerve in its longitudinal course. By convention, the ONSD was evaluated, three millimeters ( $3 \mathrm{~mm}$ ) below the papilla. At that level, the distance from the external edge to the external edge of the hyperechogenic area around the optic nerve was measured perpendicularly and corresponds to the sheath of the optic nerve, Figure 3. 
Measurement of the Optic Nerve Sheath Diameter and Transcranial Doppler Ultrasound as an Approach to Cerebral Hemodynamics

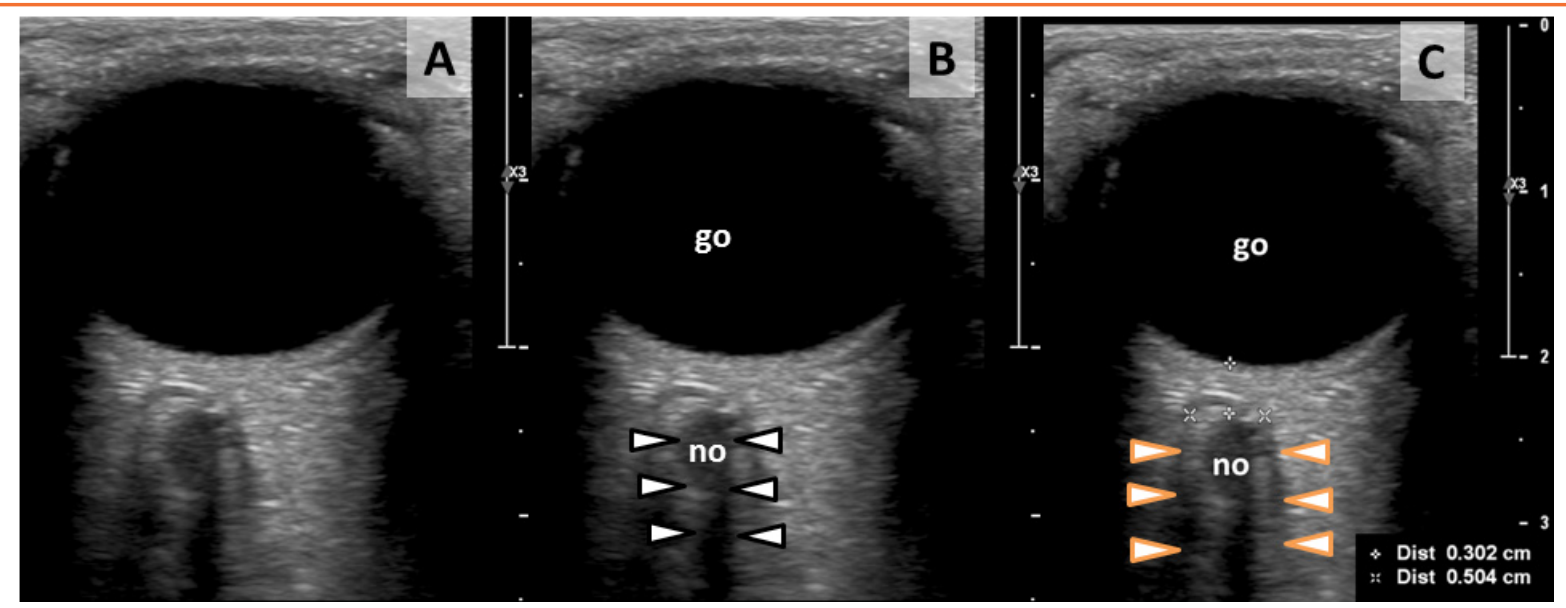

Fig 3. Retrobulbar ultrasound for measuring of the optic nerve sheath diameter (ONSD).A Conventional image. B go: eyeball, no: optic nerve, corresponds to the anechoic zone; signaled by white arrows. $C$ Methodology for measuring ONSD: a 3-mm measurement is made from the lower edge of the papilla to the optic nerve (no). At that level, the distance from the outer edge to the outer edge of the hyperechogenic area around the optic nerve is measured perpendicularly, and it corresponds to the sheath of the optic nerve (no), it is indicated by white arrows. In $C$, a first measurement of $0.30 \mathrm{~cm}$ is shown, corresponding to $3 \mathrm{~mm}$ and a second measurement of $0.50 \mathrm{~cm}$ equivalent to $5.0 \mathrm{~mm}$, which in this case corresponds to aONSD in the normal range.

\section{Statistic Analysis}

It was done with the statistical package SPSS 20 for Windows. The numerical variables were presented as absolute and average numbers with standard deviation, and limit values. The qualitative variables were presented in frequency and by hundreds. The comparison of means was made by $\mathrm{T}$ test. To evaluate the relationship between ICP measurements, mean cerebral artery mean velocity (mvMCA) by TCD and
ONSD, contingency tables with chi square were used and a bivariate correlation with determination the correlation coefficient (r) of Pearson was applied. Statisticalsignificancewasevaluatedwith $\mathrm{p}<0.05$.

\section{RESULTS}

The mean values and limit values of ONSD according to the dichotomous division of simultaneous value of ICP are shown in Table 2.

Table 2. Mean values and limits of the optic nerve sheath diameter (ONSD) according to dichotomous values of intracranial pressure (ICP).

\begin{tabular}{|c|c|c|c|c|}
\hline ICP & n (\%) & ONSDmean & Standard deviation & ONSD limits \\
\hline ICP $<20 \mathrm{mmHg}$ & $8(50 \%)$ & $5,2 \mathrm{~mm}$ & $\pm 1,3$ & $3,10-6,40 \mathrm{~mm}$ \\
\hline $\mathrm{ICP} \geq 20 \mathrm{mmHg}$ & $8(50 \%)$ & $7,4 \mathrm{~mm}$ & $\pm 0,8$ & $6,50-8,40 \mathrm{~mm}$ \\
\hline $\mathrm{p}=0,001$ & \multicolumn{3}{|l}{} \\
\hline
\end{tabular}

The value of ONSD above which there was ICP $\geq 20$ Table 3 shows the relationship between ICP and ONSD $\mathrm{mmHg}$ was $6.50 \mathrm{~mm}$, constituting the cut-off point. in a dichotomous way for each variable.

Table 3. Relationship intracranial pressure (ICP) and the optic nerve sheath diameter (ONSD) according to dichotomous values

\begin{tabular}{|c|c|c|c|}
\hline & ICP $<\mathbf{2 0} \mathbf{~} \mathbf{m H g}$ & ICP $\geq \mathbf{2 0} \mathbf{~ m m H g}$ & Total \\
\hline ONSD $<6,5 \mathrm{~mm}$ & $\mathbf{8}$ & $\mathbf{0}$ & 8 \\
\hline ONSD $\geq 6,5 \mathrm{~mm}$ & $\mathbf{0}$ & $\mathbf{8}$ & 8 \\
\hline Total & 8 & 8 & 16 \\
\hline $\mathrm{p}<0,001$ & \multicolumn{2}{|}{} \\
\hline
\end{tabular}

The correlation between ICP and ONSD was moderate and without statistical significance, it is shown in Graph 1. 
Measurement of the Optic Nerve Sheath Diameter and Transcranial Doppler Ultrasound as an Approach to Cerebral Hemodynamics

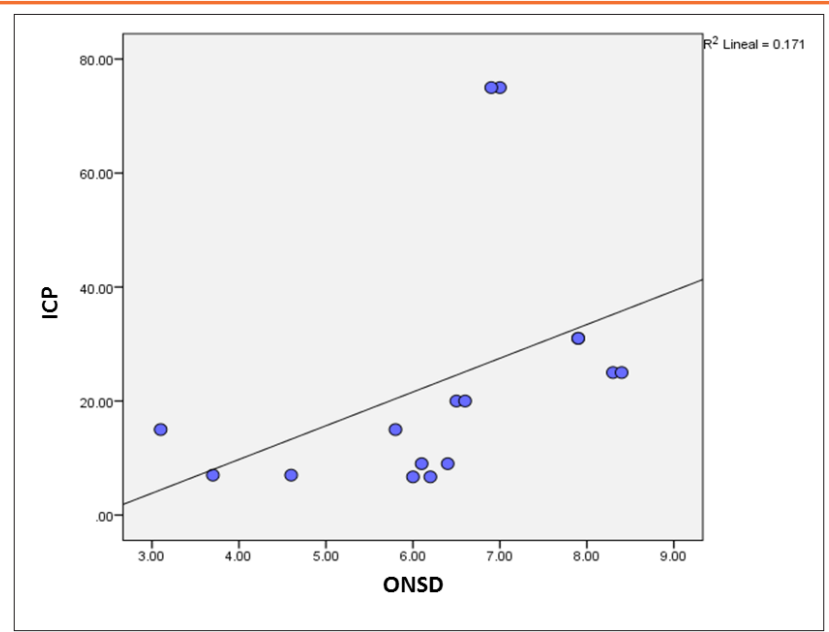

Graph 1. Correlation of intracranial pressure (ICP) and the optic nerve sheath diameter (ONSD). $r=0.414 ; p=0.111$. The mean values of mvMCA according to the dichotomous division of ONSD simultaneous value are shown in Table 4.

Table 4. Mean values of mean velocity in the right middle cerebral artery (mvMCA) according to dichotomous values of the optic nerve sheath diameter (DVNO).

\begin{tabular}{|c|c|c|c|}
\hline ONSD & n (\%) & mvMCAmean & Standard deviation \\
\hline ONSD $<6,50 \mathrm{~mm}$ & $8(50 \%)$ & $64,5 \mathrm{~cm} / \mathrm{s}$ & $\pm 9,9$ \\
\hline ONSD $\geq 6,50 \mathrm{~mm}$ & $8(50 \%)$ & $32,0 \mathrm{~cm} / \mathrm{s}$ & $\pm 12,6$ \\
\hline $\mathrm{p}<0,001$ &
\end{tabular}

The correlation between mvMCA and ONSD was moderate and inverse, with statistical significance, shown in Graph 2.

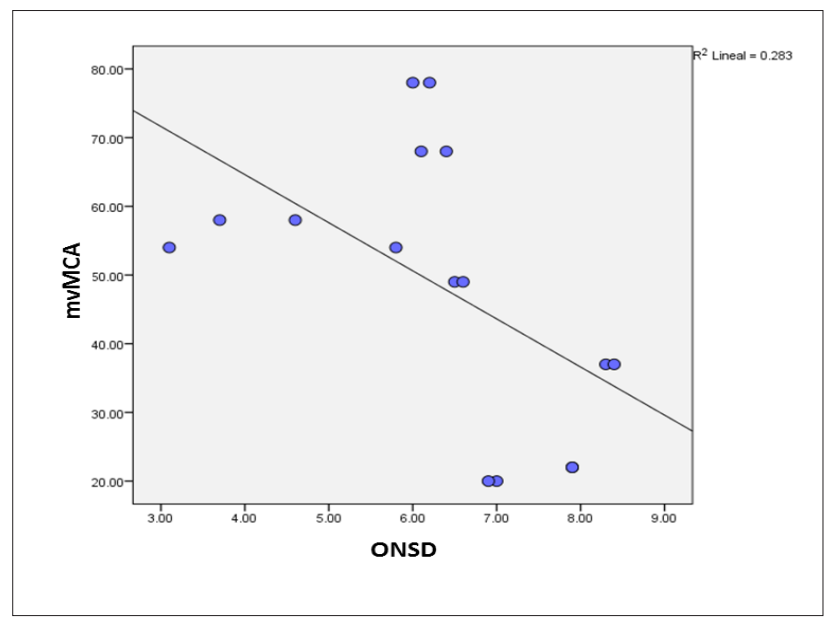

Graph 2. Mean velocity right medial cerebral artery correlation (mvMCA) and the optic nerve sheath diameter (ONSD). $r=-0.532 ; p=0.034$.

The mean values of PI according to the dichotomous division of ONSD simultaneous value are shown in Table 5

Table 5. Mean values of pulsatility index (PI) according to dichotomous values of the optic nerve sheath diameter (ONSD).

\begin{tabular}{|c|c|c|c|}
\hline ONSD & n (\%) & PImean & Standard deviation \\
\hline ONSD $<6,50 \mathrm{~mm}$ & $8(50 \%)$ & 0,8 & \pm 0.1 \\
\hline ONSD $\geq 6,50 \mathrm{~mm}$ & $8(50 \%)$ & 1,7 & $\pm 1,1$ \\
\hline $\mathrm{p}=0,038$
\end{tabular}


Measurement of the Optic Nerve Sheath Diameter and Transcranial Doppler Ultrasound as an Approach to Cerebral Hemodynamics

The correlation between PI and ONSD was scarce and without statistical significance, it is shown in Graph 3.

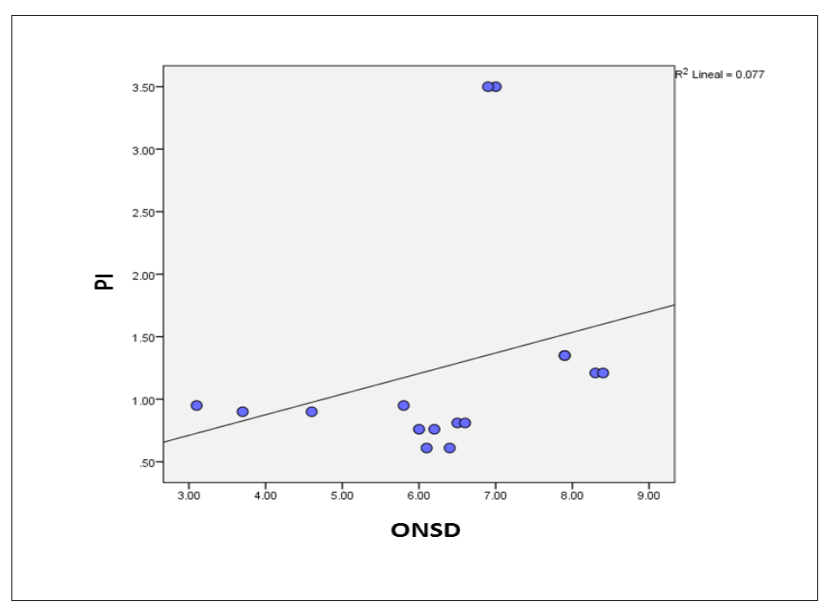

Graph 3. Pulsatibility index correlation (PI) and the optic nerve sheath diameter (ONSD). $r=0.277 ; p=0.298$.

Table 6 shows the relationship between ONSD hemodynamic patterns in the patients studied. in a dichotomous manner and simultaneous

Table 6. Relationship between cerebral hemodynamic patterns by transcranial Doppler ultrasound and dichotomous values of the optic nerve sheath diameter (ONSD).

\begin{tabular}{|c|c|c|c|c|}
\hline \multirow{2}{*}{} & \multicolumn{3}{|c|}{$\begin{array}{c}\text { Cerebral hemodynamic patterns by transcranial Doppler } \\
\text { ultrasound }\end{array}$} & \multirow{2}{*}{ Total } \\
\cline { 2 - 5 } & Normal & Hyperemia & Hypoperfusion & 8 \\
\hline ONSD $<6,50 \mathrm{~mm}$ & 4 & 4 & 0 & 8 \\
\hline ONSD $\geq 6,50 \mathrm{~mm}$ & 2 & 0 & 6 & 16 \\
\hline Total & 6 & 4 & 6 & \\
\hline $\mathrm{p}=0,005$ & \multicolumn{4}{|l}{} \\
\hline
\end{tabular}

\section{DISCUSSION}

The possibility of estimating an increase in ICP by a non-invasive method is of value for neurocritical care units. The technique from the ultrasound measurement of ONSD seems promising, however, it is necessary to homogenize it, find the cut-off points according to the study population and assess the effect of gender and anthropometric data on the reference values [8].

In the present investigation, it was important to find an ONSD cut-off point corresponding to ICP ?20 $\mathrm{mmHg}$, at a value of $6.50 \mathrm{~mm}$; above the reference value according to most of the literature reviewed.

The methodological variation that exists in the literature for the measurement of ONSD should be highlighted. Some researchers used the measurement method known as "black stripe", and measurements were made in the dark area behind the eyeball, which corresponds to the optic nerve. The current methodological criteria only accept the measurement, from external edge to outer edge of the hyperechogenic area around the optic nerve and that corresponds to the optic nerve sheath [9]. The images must be of quality, with good delimitation of the structures and the measurements in axial plane should not be compared with those made in the sagittal plane. Soliman et al [10], with a similar methodology to that used in the present investigation, determine a corresponding ONSD cut-off point with ICP $\geq$ $20 \mathrm{mmHg}$, in a value of $6.40 \mathrm{~mm}$; and for ICP $\geq 25$ $\mathrm{mmHg}$, in value of $6.60 \mathrm{~mm}$. Through an adequate statistical design the authors arrived at results that support those that are exposed in this article. The correlation analysis integrated to the previous one allows us to affirm that an ONSD $\geq 6.50 \mathrm{~mm}$ corresponds to a ICP value $\geq 20 \mathrm{mmHg}$, without a linear relationship that allows us to estimate a specific value.

The incorporation of another method of neuro monitoring with the possibility of new variables that 
can be integrated into non-invasive multiparametric monitoring, such as transcranial Doppler ultrasound with measurements of mvMCAand PI was evaluated and correlated with the ONSD measurement. It should be noted that the Doppler spectrogram can be obtained either by the conventional or blind method, with a specific equipment or by means of an ultrasound scanner with color-coded transcranial duplex availability, a possibility that can be used from the same equipment that can be used in all benefits of critical ultrasound, the variables of ONSD, mvMCA and PI are obtained, only with the change of transducers[11].

The values of mvMCA were presented in an inversely proportional relationship to the ONSD, with significant correlation. The analysis of the ONSD relationship with patterns of cerebral hemodynamics by TCD showed that all patients with cerebral hypoperfusion pattern had ONSD $\geq 6.50 \mathrm{~mm}$ and all those with hyperemic pattern had ONSD $<6.50 \mathrm{~mm}$. Recent studies that have correlated ICP measurements with variables obtained by TCD have obtained promising results. Rasulo et al demonstrated the utility of estimating ICP through TCD (ICPtcd) to diagnose intracranial hypertension. The formulas used were the following: ICPtcd $=$ Mean arterial pressure (MAP) - Estimated cerebral perfusion pressure (eCPP); eCPP=MAP $\mathrm{x}$ Diastolic velocity MCA (dvMCA) / mvMCA + 14. Robba et al [12], investigated the utility of measurements of non-invasive variables by ultrasound methods: measurement of ONSD, velocity of systolic flow in the venous rectum sinus by transcranial venous Doppler ultrasound (svVRS), and mvMCA, diastolic flow rate MCA and PI by Doppler ultrasound. The methods for estimating ICP, was correlated through invasive measurement. The superiority of the ONSD measurement and the contribution that the Doppler venous ultrasound evaluation can provide to measure sV VRS was of interest.

Withoutbeingobjective of thepresentwork, theauthors consider it useful to comment the experience with the evaluation through transcranial Doppler ultrasound of the venous spectogram. Occasional finding in the evaluation of critical patients; in neurocritical patients with elevated ICP and intracranial hypertension it becomes a frequent finding a venous sinus in which a "systolic peak" can be differentiated. Their finding and a svVRS greater than $6 \mathrm{~cm} / \mathrm{s}$ have been correlated with elevated ICP.

The results of the present investigation should be evaluated as preliminary of a study in a small series of cases. A national multicenter study could be useful in the evaluation of non-invasive multiparameter neuromonitoring with the inclusion of ONSD measurement and arterial and venous transcranial Doppler ultrasound.

In preliminary conclusions we can affirm that the ONSD finding $\geq 6.50 \mathrm{~mm}$ is indicative of ICP $\geq 20$ $\mathrm{mmH}$

\section{REFERENCES}

[1] Lazaridis C, Robertson CS. The Role of Multimodal Invasive Monitoring in Acute Traumatic Brain Injury. NeurosurgClin N Am. 2016 Oct; 27(4): 509-17.

[2] Kawoos U, McCarron RM, Auker CR, Chavko M. Advances in Intracranial Pressure Monitoring and Its Significance in Managing Traumatic Brain Injury. Int J Mol Sci. 2015 Dec 4; 16(12): 28979-97.

[3] Vaquero J, Fontana RJ, Larson AM, Bass NM, Davern TJ, Shakil AO, et al. Complications and use of intracranial pressure monitoring in patients with acute liver failure and severe encephalopathy. Liver Transpl. 2005 Dec; 11(12): 1581-9.

[4] Liu D, Li Z, Zhang X, Zhao L, Jia J, Sun F, et al. Assessment of intracranial pressure with ultrasonographicretrobulbar optic nerve sheath diameter measurement. BMC Neurol. 2017 Sep 29; $17(1): 188$

[5] Hansen HC, Helmke K. Validation of the optic nerve sheath response to changing cerebrospinal fluid pressure: ultrasound findings during intrathecal infusion tests. J Neurosurg. 1997 Jul; 87(1):34-40

[6] Koziarz A, Sne N, Kegel F, Alhazzani W, Nath S, Badhiwala JH, et al. Optic nerve sheath diameter sonography for the diagnosis of increased intracranial pressure: a systematic review and meta-analysis protocol. BMJ Open. 2017 Aug 11; 7(8): e016194.

[7] Abdo A, Pérez-Bernal J, Hinojosa R, Porras F, Castellanos R, Gómez F, et al. Cerebral Hemo 
Measurement of the Optic Nerve Sheath Diameter and Transcranial Doppler Ultrasound as an Approach to Cerebral Hemodynamics

dynamics Patterns by Transcranial Doppler in Patients With Acute Liver Failure. Transplant Proc. 2015 Nov; 47(9): 2647-9.

[8] Robba C, Santori G, Czosnyka M, Corradi F, Bragazzi N, Padayachy L, et al. Optic nerve sheath diameter measured sonographically as noninvasive estimator of intracranial pressure: a systematic review and meta-analysis. Intensive Care Med. 2018 Aug; 44(8):1284-1294.

[9] Toscano M, Spadetta G, Pulitano P, Rocco M, Di Piero V, Mecarelli O, et al. Optic Nerve Sheath Diameter Ultrasound Evaluation in Intensive Care Unit: Possible Role and Clinical Aspects in Neurological Critical Patients' Daily Monitoring. Biomed Res Int. 2017;2017:1621428.
[10] Soliman I, Johnson GGRJ, Gillman LM, Zeiler FA, Faqihi F, Aletreby WT, et al. New Optic Nerve Sonography Quality Criteria in the Diagnostic Evaluation of Traumatic Brain Injury. Crit Care Res Pract. 2018 Apr 30;2018:3589762

[11] Blanco P, Abdo-Cuza A. Transcranial Doppler ultrasound in neurocriticalcare. J Ultrasound. 2018 Mar; 21(1):1-16.

[12] Robba C, Cardim D, Tajsic T, Pietersen J, Bulman $\mathrm{M}$, Donnelly J, et al. Ultrasound non-invasive measurement of intracranial pressure in neuro intensive care: A prospective observational study. PLoS Med. 2017 14(7): e1002356.

Citation: Anselmo A. Abdo-Cuza, Roberto Castellanos-Gutierrez, Juan A. Gutiérrez-Martínez, Juliette Suárez-López, et. al. Measurement of the Optic Nerve Sheath Diameter and Transcranial Doppler Ultrasound as an Approach to Cerebral Hemodynamics. Archives of Emergency Medicine and Intensive Care. 2019; 2(1): 22-29.

Copyright: (C) 2019 Anselmo A. Abdo-Cuza, Roberto Castellanos-Gutierrez, Juan A. Gutiérrez-Martínez, Juliette Suárez-López, et. al. This is an open access article distributed under the Creative Commons Attribution License, which permits unrestricted use, distribution, and reproduction in any medium, provided the original work is properly cited. 